\title{
Application of Time Reversal to Imaging Electrical Properties of Inhomogeneous Cylindrical Objects
}

\author{
Toshifumi Moriyama and Takashi Takenaka \\ Department of Electrical and Electronic Engineering, Graduate School of Engineering, Nagasaki University \\ Nagasaki 852-8521, Japan
}

\begin{abstract}
A time-domain inverse scattering approach using time-reversed fields for reconstructing electrical parameters of cylindrical objects has been proposed in a previous work. The approach does not require the explicit knowledge of incident fields illuminating the object. The cost functional represented in terms of the time-reversed fields was minimized by a genetic algorithm (GA). In this paper, we propose a gradient-based minimization for reconstruction of an inhomogeneous object for which GA can not be applied due to computational complexity. The time-reversed fields are calculated by time-reversing the equivalent current sources determined by the total fields recorded on a measurement line. Numerical simulations are carried out to show the effectiveness of the proposed method.
\end{abstract}

Index Terms-inverse scattering, time-reversed field, microwave imaging

\section{INTRODUCTION}

Inverse scattering problems aim to determine the shape, location, and profiles of material parameters of unknown objects from the measured total fields. They have many practical applications in the fields of geophysical exploration, nondestructive testing, medical imaging, etc. Recently, timereversal (TR) techniques have been used for these applications. The TR technique was first proposed for acoustic waves [1] and later has also been applied to the electromagnetic waves [2-3]. The main feature of the TR technique is to detect a point target or a point-like target due to the spatial focusing of the time-revered wave around the target location. However, it is expected that TR can be applied to reconstruction of electrical parameters of scattering objects. We have proposed a timedomain inverse scattering method using time-reversed fields based on the field equivalent principle [4]. The time-reversed fields are calculated by time-reversing the equivalent sources corresponding to the measured total fields. The field equivalent principle shows that the field in the exterior region produced by the equivalent sources is null if and only if the medium in the interior region is the same as in the original problem. The cost functional defined in terms of the field in the exterior region was introduced and its minimization by a genetic algorithm (GA) was applied to the nondestructive testing problem of finding the location and size of unknown homogeneous defects. In case of imaging the spatial profile of electrical parameters of inhomogeneous objects, the number of unknowns becomes very large so that GA may not be an appropriate optimization tool because of the computational complexity and more efficient optimization methods are desirable.

In this paper, we apply a gradient method to the timedomain inverse scattering approach using time-reversal for reconstructing electrical parameters of inhomogeneous objects without explicit knowledge of incident fields. The explicit expression of the gradient of the cost functional of the electrical parameter functions characterizing the objects is derived. In order to confirm the effectiveness of the proposed method, numerical simulations are carried out.

\section{FORMULATION}

\section{A. Direct problem}

Let us consider the scattering by an inhomogeneous object embedded in a homogeneous background medium as shown in Fig.1(a). For simplicity, we consider two dimensional TM wave case where an electric field is parallel to the axis of the unknown cylindrical object. A short pulsed wave is generated by an electric current source $\mathbf{J}(\mathbf{r}, t)=J_{z}(\mathbf{r}, t) \hat{z}$ where $\mathbf{r}=(x, y)$ and $\hat{z}$ is the unit vector in the $z$ direction. We assume that the transmitter source is turned on at time $t=0$ and there is no electromagnetic field before $t=0$. Then, the total fields $\mathbf{v}(\mathbf{r}, t)$ satisfy Maxell's equations in the following matrix form:

$$
\left[\bar{A} \frac{\partial}{\partial x}+\bar{B} \frac{\partial}{\partial y}-\bar{C} \frac{\partial}{\partial(c t)}-\bar{D}\right] \mathbf{v}=\mathbf{j}
$$

where

$$
\begin{aligned}
& \mathbf{v}=\left(\begin{array}{c}
v_{1}(\mathbf{r}, t) \\
v_{2}(\mathbf{r}, t) \\
v_{3}(\mathbf{r}, t)
\end{array}\right)=\left(\begin{array}{c}
E_{z}(\mathbf{r}, t) \\
\eta H_{x}(\mathbf{r}, t) \\
\eta H_{y}(\mathbf{r}, t)
\end{array}\right) \quad(2 \mathrm{a}) \quad \mathbf{j}=\left(\begin{array}{c}
\eta J_{z}(\mathbf{r}, t) \\
0 \\
0
\end{array}\right) \\
& \bar{A}=\left(\begin{array}{lll}
0 & 0 & 1 \\
0 & 0 & 0 \\
1 & 0 & 0
\end{array}\right) \\
& \bar{B}=\left(\begin{array}{ccc}
0 & -1 & 0 \\
-1 & 0 & 0 \\
0 & 0 & 0
\end{array}\right) \\
& \bar{C}=\left(\begin{array}{ccc}
\varepsilon_{r}(\mathbf{r}) & 0 & 0 \\
0 & \mu_{r}(\mathbf{r}) & 0 \\
0 & 0 & \mu_{r}(\mathbf{r})
\end{array}\right)(2 \mathrm{e}) \quad \bar{D}=\left(\begin{array}{ccc}
\eta \sigma(\mathbf{r}) & 0 & 0 \\
0 & 0 & 0 \\
0 & 0 & 0
\end{array}\right)
\end{aligned}
$$


under the initial condition of zero fields $(\mathbf{v}(\mathbf{r}, t)=0, t<0)$. The parameters $\varepsilon_{r}(\mathbf{r}), \mu_{r}(\mathbf{r}), \sigma(\mathbf{r})$ are the relative permittivity, relative permeability, and conductivity of the medium, respectively. $c\left(=1 / \sqrt{\varepsilon_{b} \mu_{b}}\right)$ and $\eta\left(=\sqrt{\mu_{b} / \varepsilon_{b}}\right)$ are light speed and intrinsic impedance of the background media with permittivity $\varepsilon_{b}$, permeability $\mu_{b}$, and conductivity $\sigma_{b}=0$.

We consider two regions which are denoted by $\Omega$ and $\bar{\Omega}$ as shown in Fig.1(a). They are separated by a measurement surface $\partial \Omega$. The impressed source $\mathbf{J}$ is assumed to be in the exterior region $\bar{\Omega}$, while a scattering object is in the interior region $\Omega$. The total fields $(\mathbf{E}, \mathbf{H})$ are measured on $\partial \Omega$ during the measurement time from $t=0$ to $T$. By using the measured total fields (E, H), we can set up an interior equivalent problem where the electric and magnetic surface current sources given by $\mathbf{J}_{s}=\hat{n} \times \mathbf{H}, \mathbf{M}_{s}=\mathbf{E} \times \hat{n}$ (the unit vector $\hat{n}$ is inward normal to $\partial \Omega$ ) reproduce the same field in $\Omega$ and null field in $\bar{\Omega}$ [5].

\section{B. Time-reversed field}

Provided that the total fields inside the region $\Omega$ are negligibly small after the time $t=T$, we can set up another interior equivalent problem which is the same as we described in the previous subsection except that the problem is solved backward in time. The time-reversed field $\mathbf{u}(\mathbf{r}, t)$ of the problem is the solution of the following equations:

$$
\left[\bar{A} \frac{\partial}{\partial x}+\bar{B} \frac{\partial}{\partial y}-\bar{C} \frac{\partial}{\partial(c t)}-\bar{D}\right] \mathbf{u}=\mathbf{s}
$$

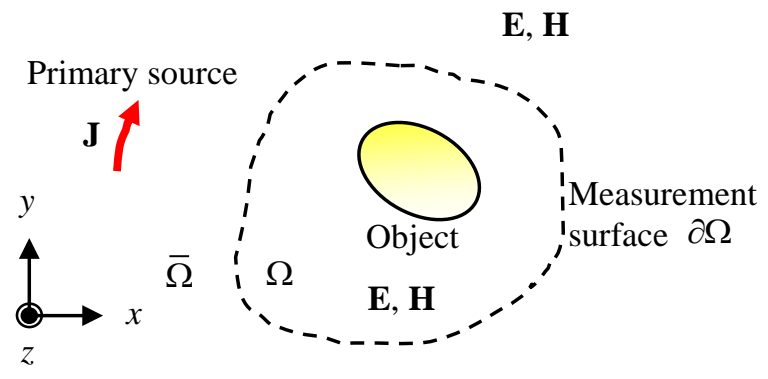

(a)

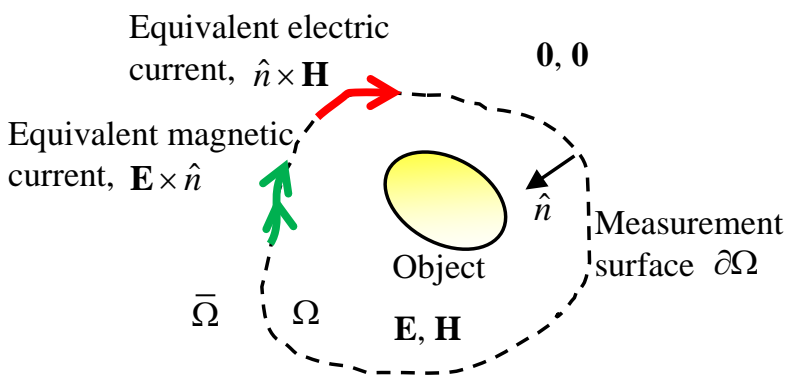

(b)

Figure 1. Geometry of the problem. (a) Original problem. (b) Equivalent problem for the interior region $\Omega$. where

$$
\mathbf{u}=\left(\begin{array}{c}
u_{1}(\mathbf{r}, t) \\
u_{2}(\mathbf{r}, t) \\
u_{3}(\mathbf{r}, t)
\end{array}\right), \quad \text { (4a) } \quad \mathbf{s}=\left(\begin{array}{c}
n_{x} \eta H_{y}-n_{y} \eta H_{x} \\
-n_{y} E_{z} \\
n_{x} E_{z}
\end{array}\right) \delta_{\partial \Omega} .
$$

under the final condition of zero fields $(\mathbf{u}(\mathbf{r}, t)=0, t>T)$. Here, $\delta_{\partial \Omega}$ is a delta function representing a source concentrated on the surface $\partial \Omega$. The source $\mathbf{s}$ produces the time reversed fields which are identical to the total fields of the original problem in $\Omega$, while null field in $\bar{\Omega}$, i.e,

$$
\mathbf{u}(\mathbf{r}, t)=\left\{\begin{array}{ll}
\mathbf{v}(\mathbf{r}, t) & \text { if } \mathbf{r} \in \Omega \\
0 & \text { if } \mathbf{r} \in \bar{\Omega}
\end{array} .\right.
$$

In the equivalent problem, the primary source $\mathbf{j}(\mathbf{r}, t)$ is not needed to calculate the time-reversed fields $\mathbf{u}(\mathbf{r}, t)$. The timereversed fields are calculated backward in time from $t=T$ to 0 .

\section{Inverse problem}

The time-domain inverse scattering problem is considered for estimating $\varepsilon_{r}(\mathbf{r}), \mu_{r}(\mathbf{r})$ and $\sigma(\mathbf{r})$ with the knowledge of the tangential components of the original total fields $\mathbf{v}(\mathbf{r}, t)$ measured on the surface $\partial \Omega$ but without explicit knowledge of the incident field. If the estimated electrical parameter is identical with true ones, the time-reversed fields cancel out the fields scattered by the object in $\bar{\Omega}$ during the interval $[0, T]$ as represented by eq.(5). On the other hand, an incorrectly estimated electrical parameter does not give null field in $\bar{\Omega}$. Based on this fact, we reduce the inverse problem discussed here to an optimization problem in which the following cost functional is minimized,

$$
Q(\mathbf{p})=\sum_{n=1}^{N} \int_{0}^{c T} \int_{\Omega} K(t)\left|\mathbf{u}_{n}(\mathbf{p} ; \mathbf{r}, t)\right|^{2} d \mathbf{r} d(c t)
$$

where $\mathbf{p}=\left[\varepsilon_{r}(\mathbf{r}), \mu_{r}(\mathbf{r}), \eta \sigma(\mathbf{r})\right]^{\mathrm{T}}$ is a vector of the electrical parameters of the object. The superscript $\mathrm{T}$ denotes the transpose. The time factor $K(t)$ is a non-negative weighting function which takes a value of zero at $t=0 . N$ successive illuminations are assumed to explore the object. The vector $\mathbf{u}_{\mathrm{n}}(\mathbf{p} ; \mathbf{r}, t)$ represents the time-reversed fields for an estimated parameter vector $\mathbf{p}$ due to the $n$-th equivalent surface current $\mathbf{s}_{\mathrm{n}}$ $\mathrm{n}=1, \ldots \mathrm{N}$.

We apply a gradient method to minimization of the cost functional. In order to get the explicit expression of the gradient, we derive the Fréchet derivative of the functional which is given by

$$
Q^{\prime}(\mathbf{p}) \delta \mathbf{p}=2 \sum_{n=1}^{N} \int_{0}^{c T} \int_{\Omega} K(t)\left[\mathbf{u}_{n}(\mathbf{p} ; \mathbf{r}, t)\right]^{\mathrm{T}} \delta \mathbf{u}_{n}(\mathbf{p} ; \mathbf{r}, t) d \mathbf{r} d(c t)
$$

where $\delta \mathbf{p}$ is a variation of the parameter $\mathbf{p}$ and $\delta \mathbf{u}_{\mathrm{n}}(\mathbf{p} ; \mathbf{r}, t)$ is the Fréchet differential of the nonlinear operator which maps the parameter vector $\mathbf{p}$ to the field vector $\mathbf{u}_{\mathrm{n}}(\mathbf{p} ; \mathbf{r}, t)$. Since the Fréchet derivative $Q^{\prime}(\mathbf{p})$ is a bounded linear functional, Riesz representation theorem says that the Fréchet differential 
$Q^{\prime}(\mathbf{p}) \delta \mathbf{p}$ is represented as an inner product of $\delta \mathbf{p}$ and the gradient vector $\mathbf{g}$.

$$
Q^{\prime}(\mathbf{p}) \delta \mathbf{p}=\langle\mathbf{g}(\mathbf{r}), \delta \mathbf{p}(\mathbf{r})\rangle
$$

where the inner product of three-dimensional vector functions is defined by

$$
\langle\mathbf{a}(\mathbf{r}), \mathbf{b}(\mathbf{r})\rangle=\sum_{i=1}^{3} \int_{\bar{\Omega}} a_{i}(\mathbf{r}) b_{i}(\mathbf{r}) d \mathbf{r}
$$

Following a similar way described in the reference [6], the gradient of the functional (6) is given by

$$
\mathbf{g}(\mathbf{r})=\left[g_{\varepsilon}(\mathbf{r}), g_{\mu}(\mathbf{r}), g_{\sigma}(\mathbf{r})\right]^{\mathrm{T}}
$$

where

$$
\begin{aligned}
& g_{\varepsilon}(\mathbf{r})=\int_{0}^{c T} \sum_{n=1}^{N} w_{1, n}(\mathbf{p} ; \mathbf{r}, t) \frac{\partial u_{1, n}(\mathbf{p} ; \mathbf{r}, t)}{\partial(c t)} d(c t) \\
& g_{\mu}(\mathbf{r})=\int_{0}^{c T} \sum_{m=2}^{3} \sum_{n=1}^{N} w_{m, n}(\mathbf{p} ; \mathbf{r}, t) \frac{\partial u_{m, n}(\mathbf{p} ; \mathbf{r}, t)}{\partial(c t)} d(c t) \\
& g_{\sigma}(\mathbf{r})=\int_{0}^{c T} \sum_{n=1}^{N} w_{1, n}(\mathbf{p} ; \mathbf{r}, t) u_{1, n}(\mathbf{p} ; \mathbf{r}, t) d(c t) .
\end{aligned}
$$

The adjoint field vector $\mathbf{w}(\mathbf{p} ; \mathbf{r}, t)$ is written as

$$
\mathbf{w}=\left(\begin{array}{l}
w_{1}(\mathbf{p} ; \mathbf{r}, t) \\
w_{2}(\mathbf{p} ; \mathbf{r}, t) \\
w_{3}(\mathbf{p} ; \mathbf{r}, t)
\end{array}\right) .
$$

This field is the solution of the following adjoint equations:

$$
\left[-\frac{\partial}{\partial x} \bar{A}-\frac{\partial}{\partial y} \bar{B}+\frac{\partial}{\partial(c t)} \bar{C}-\bar{D}\right] \mathbf{w}=2 K(t) \mathbf{u}(\mathbf{p} ; \mathbf{r}, t) \Psi(\mathbf{r})
$$

where

$$
\Psi(\mathbf{r})= \begin{cases}0 & \mathbf{r} \in \Omega \\ 1 & \mathbf{r} \in \bar{\Omega}\end{cases}
$$

under the initial condition $(\mathbf{w}(\mathbf{r}, t)=0, t<0)$. By using this gradient, the conjugate gradient method solves the minimization problem. The update formula for the unknown electrical parameters is given by

$$
\mathbf{p}^{(k+1)}(\mathbf{r})=\mathbf{p}^{(k)}(\mathbf{r})+\alpha^{(k)} \mathbf{d}^{(k)}(\mathbf{r})
$$

where the superscript $(k)$ indicates the k-th iteration. The search direction $\mathbf{d}^{(\mathrm{k}+1)}$ is computed by

$$
\mathbf{d}^{(k+1)}(\mathbf{r})=-\mathbf{g}^{(k+1)}(\mathbf{r})+\frac{\left\langle\mathbf{g}^{(k+1)}-\mathbf{g}^{(k)}, \mathbf{g}^{(k+1)}\right\rangle}{\left\langle\mathbf{g}^{(k)}, \mathbf{g}^{(k)}\right\rangle} \mathbf{d}^{(k)}(\mathbf{r})
$$

The step size $\alpha^{(\mathrm{k})}$ is determined by a line search along the search direction $\mathbf{d}^{(\mathrm{k})}$ where the functional takes a minimum.

\section{NUMERICAL RESULTS}

To confirm the effectiveness of the proposed inverse scattering method using time-reversed field based on the gradient method, numerical simulations are performed. In these examples, the synthetic measured data, time-reversed field and adjoint field are calculated by the finite-difference time-domain
(FDTD) method. The FDTD solution space consists of $180 \Delta \times$ $180 \Delta$ cells where $\Delta=\Delta x=\Delta y$ is the cell size. The primary source is given by

$$
\mathbf{J}_{n}=I(t) \delta\left(\mathbf{r}-\mathbf{r}_{n}\right) \hat{z}
$$

where $\mathbf{r}=\mathbf{r}_{n}$ is the location of the n-th primary source and its time function is expressed as

$$
I(t)=\tau \frac{d}{d t}\left[\left(\frac{t}{\tau}\right)^{4} \exp \left(-\frac{t}{\tau}\right)\right]
$$

The background is free space. The background is used as an initial guess of the reconstruction. The synthetic measured data are contaminated by additive white Gaussian noise with $\mathrm{SNR}=20 \mathrm{~dB}$. The SNR is defined by

$$
\mathrm{SNR}=10 \log _{10} \frac{\sum_{n=1}^{N} \int_{0}^{c T} \int_{\partial \Omega}\left|\mathbf{u}_{n}(\mathbf{r}, t)\right|^{2} d S d(c t)}{\sum_{n=1}^{N} \int_{0}^{c T} \int_{\partial \Omega}\left|\mathbf{n}_{n}(\mathbf{r}, t)\right|^{2} d S d(c t)}
$$

where $\mathbf{u}_{n}$ are the measured data and $\mathbf{n}_{n}$ are the additive noise.

As a first example, we consider the reconstruction of a Lshape cylinder which has the relative permittivity of $\varepsilon_{r}=2$. Twelve sources are placed on the boundary line of the $5 \lambda \times 5 \lambda$ square with the center at the origin $(x, y)=(0,0)$ and are successively used to probe the cylinder. $\lambda$ is the wavelength in free space corresponding to the highest frequency contained in the time factor of the primary source (the highest frequency is defined by the frequency at which the source spectrum is $1 / 20$ of the maximum value).The parameter of the excitation factor are $\tau=0.129$ ns so that the shortest wavelength is $\lambda=0.09 \mathrm{~m}$. The cell size and time step size is chosen to be $\Delta=0.0045 \mathrm{~m}$ and $\Delta t=10.4 \mathrm{ps}$, respectively. The time duration $T$ of the measurement is $3000 \Delta t$. For each illumination, the measurements of tangential components of the total fields are carried out along the measurement line of the $3 \lambda \times 3 \lambda$ square. The reconstruction domain is the inside area bounded by the measurement line. Figure 2(a) shows the original profile of the cylinder. The reconstruction after 100 iterations is shown in Fig. 2(b). To examine the effect of noise on the stability of the proposed method, the reconstruction with $\mathrm{SNR}=20 \mathrm{~dB}$ is shown in Fig.2(c). Both reconstructed profiles are very similar to the original profile.

Next, we consider the reconstruction of an inhomogeneous cylindrical object. The parameters of the FDTD grid and source and the measurement configuration are the same as that used in the previous example. The relative permittivity of the object is a smooth sinelike profile shown in Fig. 3(a):

$$
\varepsilon_{r}(x, y)=\left\{\begin{array}{cl}
2 \sin \left(\frac{\pi(a-r)}{2 a}\right)+1 & 0 \leq r=\sqrt{x^{2}+y^{2}} \leq a \\
1 & \text { otherwise }
\end{array}\right.
$$

where the radius $a$ of the object is $20 \Delta x$. The reconstructed results after 50 iteration without/with noise are shown in 
Fig.3(b), (c). It is found that a smooth profile is reconstructed faster than a discontinuous profile.

The third example is the reconstruction of multiple objects of 'Austria' profile enclosed by a square wall. The parameters of the time function of the primary source are $\tau=0.36 \mathrm{~ns}$ so that the shortest wavelength is $\lambda=0.254 \mathrm{~m}$. The boundary line of twelve sources is the $6.3 \lambda \times 6.3 \lambda$ square. The measurements of tangential components of the total fields are carried out along the measurement line of the $5.9 \lambda \times 5.9 \lambda$ square. The cell size and time step size is chosen to be $\Delta=0.01 \mathrm{~m}$ and $\Delta t=23.1 \mathrm{ps \text {, }}$ respectively. The time duration $T$ of the measurement is $3500 \Delta t$. The inner boundary of the wall with width $0.4 \lambda$ is a $3.9 \lambda \times$ $3.9 \lambda$ square. Its relative permittivity is 4.0 . The inside and outside of the wall is air. The 'Austria' profile objects consist of two discs and one ring. The discs of radius $0.4 \lambda$ are centered at $(1.2 \lambda, 2.4 \lambda)$ and $(-1.2 \lambda, 2.4 \lambda)$, respectively. The ring with an inner radius of $0.6 \lambda$ and an outer radius of $1.2 \lambda$ is centered at $(0,-0.8 \lambda)$. The relative permittivity of the ring and discs is 2 . Fig. 4(a) shows the true 'Austria' profile. The wall is assumed to be known. The reconstructed domain is the region interior to the wall. The reconstructed results after 500 iterations without/with noise are shown in Fig. 4(b), (c). It can be seen that the proposed method gives an accurate reconstruction and is not so sensitive to the presence of noise in the measured field data.

These results show that the proposed method is very effective.

\section{CONCULUSIONS}

A gradient-based inverse scattering approach using timerevered fields to reconstruct electrical parameters of inhomogeneous cylindrical objects without explicit knowledge of incident fields has been proposed. Numerical simulation has demonstrated the validity of our inverse technique. We have proposed another inverse scattering approach based on the field equivalence principle in a previous work [7]. We will consider a combination of both approaches.

\section{REFERENCES}

[1] M. Fink, "Time reversal in acoustics," Contemparary Physics, pp.95109, vol. 37, no.2, 1996.

[2] G. Micolau, M. Saillard, and P. Borderies, "DORT method as applied to ultrawideband signals for detection of buried objects," IEEE trans. Geosci. Remote Sensing, pp.1813-1820, vol.41, no.8, Aug, 2003.

[3] P. Kosmas and C. M. Rappaport, "A matched-filter FDTD-based time reversal approach for microwave nreast cancer detection,” IEEE trans. Anttenas Propag., pp.1257-1264, vol.54, no.4, Apr., 2006.

[4] T. Moriyama T. Tanaka and T. Takenaka, "Time reversal imaging without information of incident field," Proc. of 2013URSI International Symposium on Electromagnetic Theory (URSI-EMTS 2013), pp. 123-126, Hiroshima, Japan, May, 2013.

[5] R. F. Harrington, Time-Harmonic Electromagnetic Fields, Wiley-IEEE Press; 2nd edition, 2001.

[6] T. Tanaka, T. Takenaka and S. He, "An FDTD approach to the timedomain inverse scattering problem for an inhomogeneous cylindrical object”, Microwave Opt Technol Lett, pp.72-77, vol.20, no.1, January, 1999.

[7] T. Takenaka and T. Moriyama, "Inverse scattering approach based on the field equivalence principle: inversion without a priori information on incident fields,” Optics Letters, pp.3432-3434, vol. 37, no. 16, August, 2012

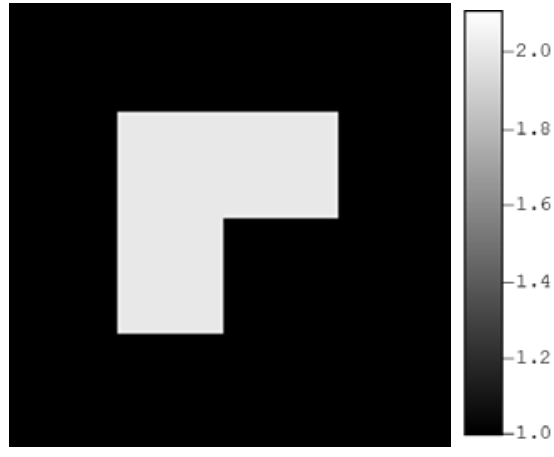

(a)

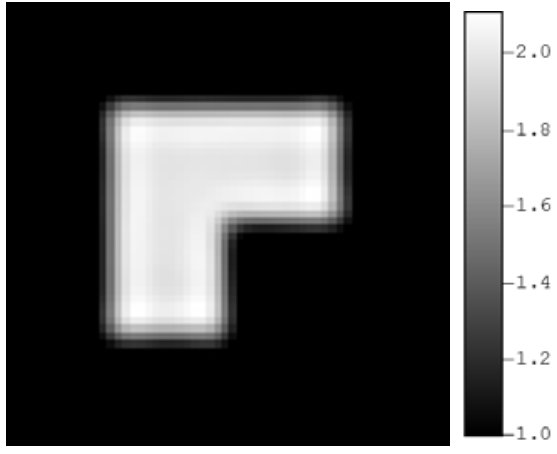

(b)

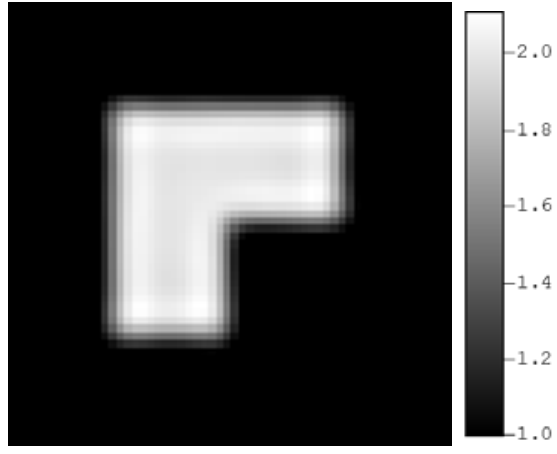

(c)

Figure 2. Reconstructed results of relative permittivity distribution of a L-shape cylinder. (a) Original profile. (b) Reconstruction after 100 iterations. (c) Reconstruction after 100 iterations with noise(SNR=20dB). 


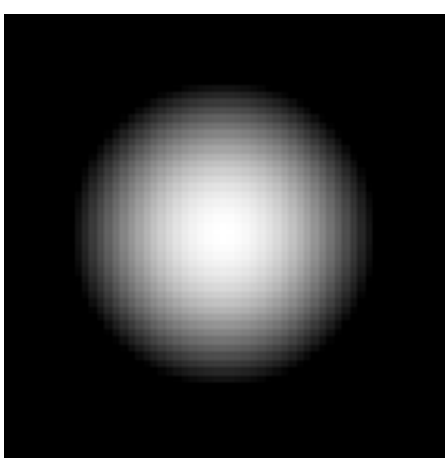

(a)

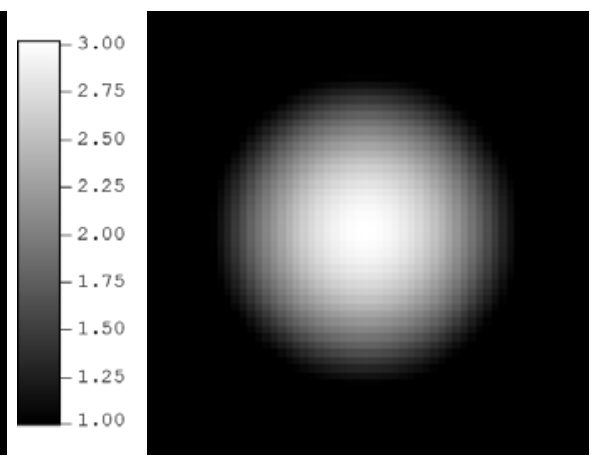

(b)

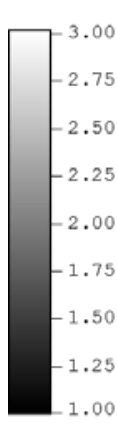$$
80
$$

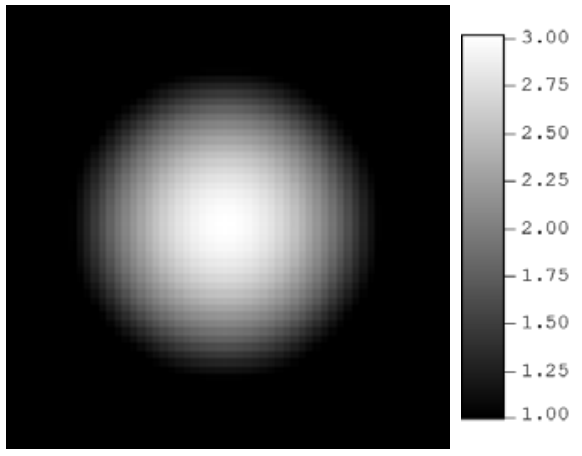

(c)

Figure 3. Reconstructed results of relative permittivity distribution of an inhomogeneous cylinder. (a) Original profile. (b) Reconstruction after 100 iterations. (c) Reconstruction after 50 iterations with noise(SNR=20dB).

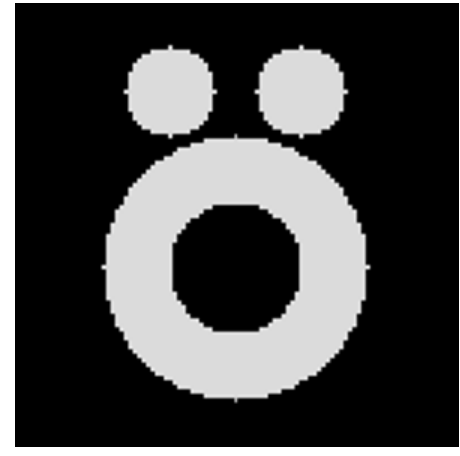

(a)

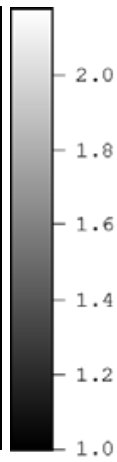

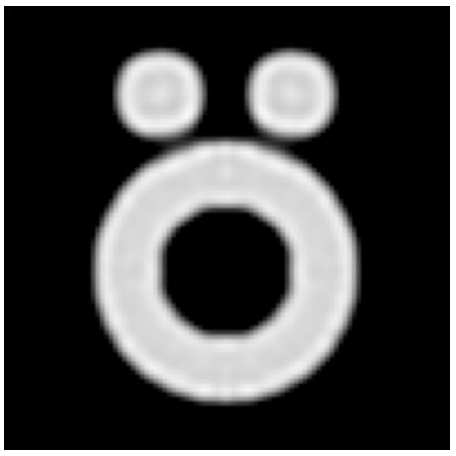

(b)

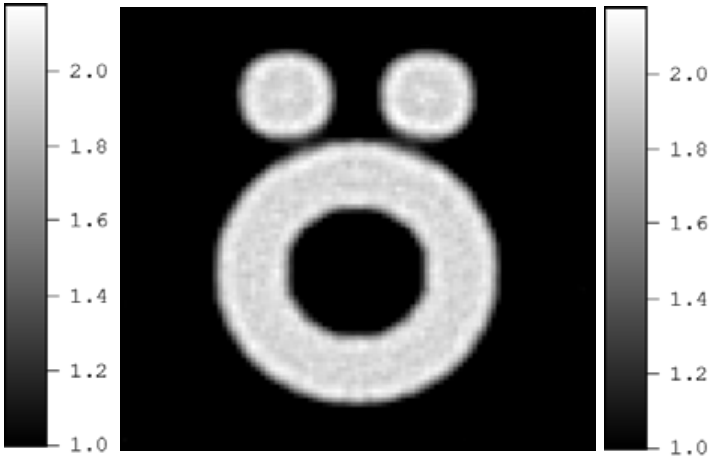

(c)

Figure 4. Reconstructed results of relative permittivity distribution of multiple objects of 'Austria' profile. (a) Original profile. (b) Reconstruction after 100 iterations. (c) Reconstruction after 100 iterations with noise(SNR=20dB). 